\title{
WHAT IS THE CONNECTION BETWEEN ELLIPTICALS AND
}

\section{BULGES?}

\author{
MARIJN FRANX \\ Harvard-Smithsonian Center for Astrophysics, 60 Garden Street, Cambridge, MA 02138
}

\begin{abstract}
The structure of bulges and ellipticals, and their relation to galaxy halos are reviewed. Since many ellipticals contain faint disks, the qualitative distinction between bulges and ellipticals is more accurately described as a quantitative variation in Bulge/Disk ratio. The exception may be the brightest ellipticals, which are usually Bright(est) Cluster Members. The available evidence suggests that the spheroid properties are determined by more than halo properties alone. This is clearest for systems with low B/D ratio, where bulge velocity dispersions are similar to disk velocity dispersions. Constraints on the stellar formation scenarios are discussed.
\end{abstract}

Key words: elliptical galaxies - bulges - dark halos - formation - stellar populations

\section{Introduction}

The idea that galaxies can be dissected into different components is fundamental to almost all classification schemes. As an example, one of the important characteristics of the Hubble sequence (Hubble 1936) is that the bulge to disk ratio increases towards early types. The underlying assumption is that the different components by themselves are very similar between galaxies, but that their relative masses within galaxies differ, and determine the Hubble type.

We would currently distinguish four different components in galaxies: the bulge or spheroid, the disk, the interstellar medium, and the dark matter halo. These four components may be present in almost all galaxies. Probably all galaxy types contain significant amounts of gas, from ellipticals which contain hot gas observed in X-rays, to late-type spirals containing large amounts of cool H I. Spiral galaxies definitely have large, massive halos, but the situation is not completely clear for ellipticals. This will be discussed below. Even disk components may be present in almost all ellipticals, except possibly, the brightest (which are in many cases also the brightest cluster members). Thus, the general separation of galaxies into these components appears to be very successful.

The next questions that one can ask is how the components compare in detail, and, more fundamentally, how they were formed. Here we focus on the properties of spheroids, (ellipticals and bulges), their relations to halos, and constraints on their formation.

\section{How simple are Spheroids?}

In the 1960's and early 70's, it was believed that spheroids were simple systems. Mass was thought to be the main parameter; the flattening was thought to be due to rotation. The stellar populations were modeled with single metallicity, single age components. The formation of spheroids was thought to have taken place in the very early universe. 

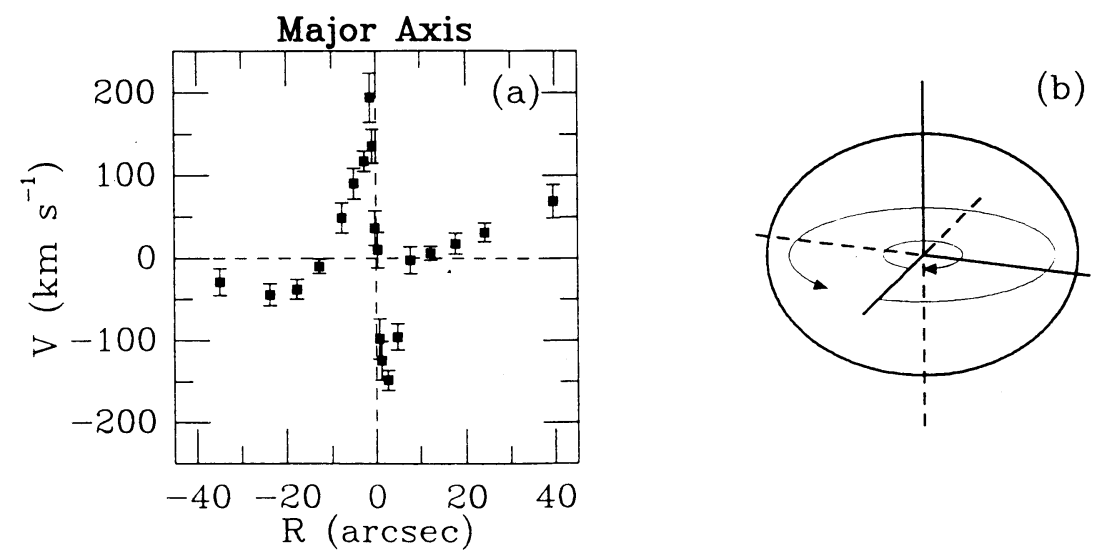

Fig. 1. The complex kinematics of IC 1459. (a) the rotational velocity along the major axis. The inner parts appear to counter-rotate compared to the outer parts. This is schematically drawn in (b). A more detailed analysis has shown that the central parts can be decomposed in to two components: one hot component, slowly rotating like the outer parts, and one cold component, rapidly counter rotating with respect to the outer parts. The cold component may not contribute more than $20 \%$ of the light, but dominates the kinematics (Franx \& Illingworth 1988).

\subsection{KINEMATICS}

New observational evidence collected over the last 2 decades has challenged this common wisdom. The conference proceedings of IAU 100 (Athanassoula 1983), and IAU 127 (de Zeeuw 1987) contain much of the new material. As an extreme example, figure 1 shows the kinematics of IC 1459, a normal, southern elliptical. This galaxy appears perfectly normal on the sky, but the kinematics show that the inner parts are counter-rotating with respect to the outer parts. A detailed spectroscopic analysis showed that the central part consists of two components, a slowly rotating component related to the bulk of the galaxy, and a rapidly, counter-rotating component, which may contribute about $20 \%$ of the light (Franx and Illingworth 1988).

Many other such systems have been found (Bender 1988, Jedrzejewski \& Schechter 1988, Franx et al 1989), including systems like NGC 4406, in which the angular momentum of the central regions is perpendicular to the angular momentum of the outer parts. The overall statistics are that about $25 \%$ of observed ellipticals show such kinematically distinct nuclei.

The formation of such systems is not known with certainty. It is likely that a merger, or accretion of gas or stars played a role. The phenomenon is possibly related to star burst galaxies, in which comparable amounts of $\mathrm{CO}$ are observed near the central parts, and which are thought to be triggered by mergers.

There is no reason to assume that the kinematically distinct cores were formed 
only recently. The two body relaxation time is so large, that the subcomponents will persist for many Hubble times. It is very well possible that the subcomponents were formed as part of the generic formation process of ellipticals. The fact that the stellar populations of the galaxies with such nuclei are normal is consistent with this hypothesis.

The relatively high rate of kinematically distinct nuclei in ellipticals poses the question whether similar nuclei exist in bulges. Some bulges in nearby galaxies (like M31, NGC 4594) contain rapidly rotating subcomponents (e.g., Dressler 1984, Kormendy 1988), but until now, no counter rotating subcomponents have been found. A survey of the kinematics of S0 galaxies has until now not produced a single such case (Fisher et al 1993).

However, Rubin has recently discovered the case of NGC 4550 in a similar survey (Rubin et al 1992). Her data showed clear evidence for two counter rotating components in this S0 galaxy. Whereas the author thought that this galaxy might be the first case of a kinematically distinct nucleus in a bulge, this turns out not to be the case. A multicomponent analysis by Rix et al (1992, see also this volume), shows that the two counter-rotating components reside in two co-spatial, cold, disks. The two disks have comparable exponential luminosity profiles, and are like normal disks. The bulge of the galaxy is not rotating at all. It is likely that the counter rotating disk was formed by the infall of counter rotating gas. Either the bulge was not rotating before the accretion took place, or during the accretion process bulge stars were added to produce a non rotating bulge.

\subsection{PHOTOMETRY}

In addition, there is photometric evidence for substructure in ellipticals. Carter (1978, 1986) detected non-elliptical isophotes in ellipticals, and later surveys showed large numbers of ellipticals with such deviations (e.g., Bender et al 1988). A significant number of those deviations are "disk-like", i.e., they can be explained by the presence of a disk. The deviations are expressed in a parameter $c_{4}$, which characterizes the residual amplitude after the ellipsefit in the $\cos 4 \theta$ harmonical. Positive $c_{4}$ indicates disky isophotes, negative $c_{4}$ indicates boxy isophotes.

The value of $c_{4}$ lies usually in the range of $-0.04 \leq c_{4} \leq 0.04$. This might suggest that any disk contributes only several percents of the light. However, an analysis by Rix and White (1990) showed that this is a serious underestimate of the required disk fraction. Because of projection effects, disks produce very small $c_{4}$ terms when the galaxy is seen face-on. When the galaxies are seen edge-on, they tend to be classified as S0, and not elliptical, because of the disk. They concluded that a large fraction of ellipticals may contain disks contributing $20 \%$ of the light.

This result is consistent with the work of others, who had speculated that many ellipticals were face-on S0's (i.e., they have disks), e.g. Capaccioli (1986), van den Bergh (1990). Complete surveys of ellipticals and S0s are needed to put strong constraints on the fraction of the light in such disks. It is interesting to note, that two independent surveys both conclude that many, if not most, low luminosity ellipticals contain disks (Capaccioli et al 1992, Rix 1993).

We conclude that both the kinematics and the photometry show that most el- 
lipticals have substructure. This significantly narrows the "gap" between ellipticals and S0's, and indicates that the formation of disks is an even more common aspect of galaxy formation than thought before. Truly disk-less ellipticals may be exceedingly rare, and those may be mainly bright(est) cluster members.

\section{Can we distinguish between Ellipticals and Bulges ?}

This issue has been reviewed extensively by Kormendy (1982), Illingworth (1983), Barnes and White (1984). Here a brief summary is given.

\subsection{STRUCTURE}

The fact by itself that it has taken more than 10 years of CCD photometry studies to decide whether or not ellipticals have substantial disks demonstrates the photometric similarity of bulges and ellipticals. Most bulges follow an $r^{1 / 4}$ law reasonably well, although some exceptions have been reported (see, e.g. Kormendy 1982). These exceptions are generally systems with low Bulge/Disk ratio. Infrared observations would be useful to minimize the influence of dust on the observed profiles.

An early analysis by Barnes and White (1984) of the data by Kent (1984) showed that the distribution of structural parameters (effective radii and surface brightness) were very similar. This important result puts tight constraints on the formation of spheroids.

\subsection{KINEMATICS}

The kinematics of bulges and ellipticals give a somewhat more confusing result. The rotational support of ellipticals correlates rather strongly with luminosity, in the sense that bright ellipticals have low rotation, and faint ellipticals (around $\mathrm{L}_{*}$ ), rotate rather rapidly (Davies et al 1983). No such trend has been observed for bulges, all bulges have been found to be rotating rapidly (e.g., Kormendy and Illingworth 1982). This is partly or fully due to the fact that it is hard to find truly bright bulges, but it remains somewhat uncomfortable that no slowly rotating bulges have been found. The only known exception is the bulge of NGC 4550, which is faint, and not rotating (Rix et al 1992). As discussed above, this galaxy has two counter rotating disk components, and no rotation is detected in the central bulge region.

The interpretation that luminosity is the driving parameter has become more uncertain with the discovery that many of the low luminosity ellipticals have disks, and that rotation correlates with isophotal shapes (Bender 1988a). Thus, when we compare low luminosity ellipticals with low luminosity bulges, we may be comparing similar galaxies at different inclinations, and not different galaxy types. It is very hard to separate the contribution of the disk to the apparent rotation, and it may be that the rotation of the spheroid in inclined systems has been overestimated.

Another complication is the result that some extremely low luminosity ellipticals show no significant rotation. Bender \& Nieto (1990) found that low surface brightness dwarfs show little rotation, demonstrating that more parameters than luminosity alone determine the rotation of galaxies. 
It has been established that elliptical galaxies satisfy relations like the Faber Jackson relation, the Fundamental Plane, and the $D_{n}-\sigma$ relation (see de Zeeuw and Franx 1991, and references therein). These are basically relations between structural parameters (effective radius, surface brightness), and velocity dispersion. Early studies gave conflicting results as to the question whether bulges follow the same relations as ellipticals. Recent results appear to indicate that this is indeed the case for the Fundamental Plane, within reasonable accuracy (Dressler 1987, Bender et al 1992). The question whether the accuracy is high enough to allcw the use of bulges as distance indicators is not completely resolved.

The origin of these relations is not quite clear. The fundamental plane relation implies that Mass to Light ratio (M/L) varies slowly with luminosity (Faber et al 1987). It is not known with certainty whether the $\mathrm{M} / \mathrm{L}$ ratio is entirely determined by the stellar population, or whether dark matter plays a role. It is not known either whether the metallicity (and thereby the $\mathrm{M} / \mathrm{L}$ ) of bulges is determined by the luminosity of the bulge, or by the total luminosity. Thus the application of these relations to bulge systems is somewhat uncertain. It appears, however, that the $\mathrm{M} / \mathrm{L}$ ratios of bulges are roughly the same as those of ellipticals.

\subsection{STATISTICAL RELATIONS}

The above suggests that it is virtually impossible to distinguish between bulges and ellipticals by a study of the spheroidal component alone. Furthermore, many ellipticals turn out to have weak disks. This suggests that the main distinction between bulges and ellipticals is Bulge to Disk ratio (B/D). The question whether differences exist between bulges and ellipticals can be rephrased into the question whether the $\mathrm{B} / \mathrm{D}$ ratio can be determined from properties of the spheroid. In the following, we address the question whether such distinction can be made in a statistical sense.

\subsubsection{Luminosity Function}

Dressler and Sandage (1983) suggested that the luminosity function of bulges and ellipticals differ. In the faint regime, bulges are more prevalent, and at the high end, ellipticals dominate. A problem with this kind of comparison is that many faint bulges reside in luminous disks, and are included in magnitude selected samples because of the bright disk. However, a detailed study of the luminosity function of Virgo galaxies by types has confirmed that ellipticals are more abundant at the high luminosity end (Binggeli, Sandage, and Tammann 1988).

Schechter \& Dressler (1987) determined B/D ratios for a complete field sample. The result is given in Figure 2. The scatter in B/D ratio is larger than the systematic trend; but the median points do indicate that the $B / D$ ratio increases slightly with luminosity. Since the B/D ratio is plotted against total luminosity, not bulge luminosity, this data supports strongly the notion that faint bulges have generally lower B/D ratios. 


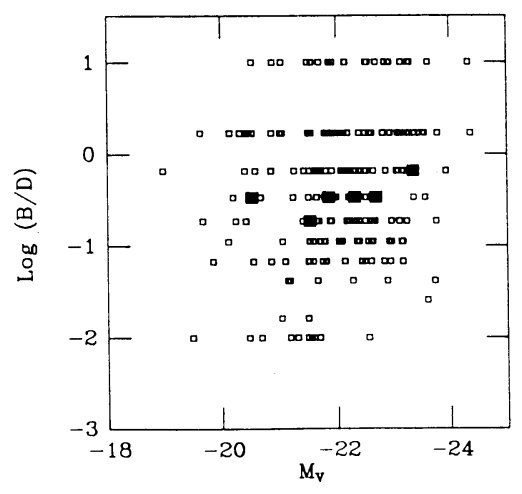

Fig. 2. The Bulge/Disk ratio plotted against total luminosity, based on the data from Schechter \& Dressler (1987). The scatter dominates the relation. The filled symbols are medians of equal sized subsamples. There is a weak relation, in the sense that brighter galaxies have somewhat larger B/D ratios.

\subsubsection{Environment}

Dressler (1980) and Schechter \& Dressler (1987) also found that the B/D ratio depends on environment, in the sense that galaxies have higher B/D ratios at higher densities. This interesting result is not well understood. It can be partly due to the fact that disk galaxies in dense environments have low starformation rates, and thus the disk $\mathrm{M} / \mathrm{L}$ ratio may be systematically higher. It is just as well possible, however, that processes at early times caused the bulge to be larger in higher densities, possibly at the cost of part of the disk. One might think of interactions with other galaxies, accretion of smaller galaxies, etc. For discussions of possible effects, see Dressler (1980), Kent (1981), Schechter \& Dressler (1987).

\section{Relation between spheroid and halo}

One of the most fundamental structural questions one can ask in the comparison of bulges and ellipticals is whether or not both are surrounded by a halo. We know that many field spirals have halos, which contribute $50 \%$ or more of the mass in the luminous region, and which continue to large radii (e.g., Broeils 1992, and references therein). Halos are no doubt the dominant component in spiral galaxies, and the formation of spirals is currently thought to be driven by the collapse of the halos. The luminous matter may not be important at all for the collapse of the proto galaxy.

An obvious question therefore is whether ellipticals, and S0's, have halos. If the answer is negative, then the process by which these galaxies formed must have been dramatically different, or they must have lost their halo later. 


\subsection{Do Ellipticals haVE halos?}

One of the easiest ways to determine the mass profile of a galaxy is by measuring the rotation curve from a gas disk. Unfortunately, there are not many ellipticals with regular gas disks. The number of known gas disks in truly luminous ellipticals is virtually zero. Thus the information is rather sparse. Kent (1990) and de Zeeuw (1992) reviewed the available data, and concluded that the H1 data is generally consistent with the hypothesis that ellipticals have halos, but the evidence is far from overwhelming. A complicating factor is that ellipticals may have triaxial shapes (e.g., Franx et al 1991), and that gas orbits may be non-circular.

It is much more difficult to use the stellar kinematics to determine the density profile of elliptical galaxies. The reason is that the velocity dispersions can be anisotropic, and that introduces an extra degree of freedom (Binney \& Mamon 1982). This makes it impossible to determine the density profile with the same accuracy as density profiles for spirals. Nevertheless, early results by Efstathiou et al (1982) indicated that the $\mathrm{M} / \mathrm{L}$ ratio of NGC 5813 increases at large radii.

One way to solve the ambiguity is to measure velocity dispersions at large distances from the center (e.g., Saglia et al 1993). Unfortunately, this becomes extremely hard, as the surface brightness decreases rapidly with radius beyond 1 effective radius $\left(I \propto r^{-2}\right)$.

Possibly the best tracer to use is the X-ray emitting hot gas, which is quite ubiquitous in luminous ellipticals (e.g., Forman et al 1985, Trinchieri et al 1986). Surface brightness and temperature measurements are necessary to obtain good density profiles. Only very few galaxies observed with the Einstein satellite had their temperature profiles measured, and thus the mass determinations were still rather uncertain. It is to be expected that the ROSAT satellite will produce accurate temperature measurements in the near future.

A more indirect argument is based on the fact that rich clusters appear to have rather high mass to light ratios. When one compares poor groups to rich clusters, the overall luminous baryonic matter to dark matter ratio appears to be roughly constant (Blumenthal et al 1984). Since the morphological mixes in groups and clusters vary widely with their density, this can be taken as proof that the ratio of dark matter per luminous baryonic matter does not change systematically with morphological type.

\subsubsection{Do we expect Ellipticals to have halos?}

Another constraint on the mass of ellipticals or S0's follows from the dynamics of clusters. Merritt (1988) analyzed the effect of tidal stripping in the center of the Coma cluster. He concluded that the ratio of matter bound to galaxies to the total matter $M_{g a l} / M_{\text {tot }} \leq 0.15$. The result implies that for individual galaxies $M_{\text {gal }}<5 M_{\text {lum }}$, where $M_{\text {gal }}$ is the total galaxy mass. This implies that the early type galaxies in the centers of clusters may have lost a significant part of their halos to the cluster. The dark matter content of galaxies may obviously be influenced by their environment. 


\subsection{HOW ARE THE HALOS RELATED TO THE LUMINOUS PARTS?}

In the following, it is assumed that early type galaxies do have halos. It is then natural to ask, how the halos of galaxies are related to the luminous parts, and whether any systematic relationships can be found. The best observed parameter of a halo of a spiral galaxy is its circular velocity. We use the circular velocity of the $\mathrm{H} \mathrm{I}$ gas as the circular velocity of the halo. For elliptical galaxies, no similar observable exists, but we can estimate circular velocities from observations of velocity dispersion profiles.

Van der Marel (1991) calculated axisymmetric models to fit observed photometry and velocity dispersions of a sample of 37 ellipticals. These models calculations were repeated by the author, with the inclusion of a dark halo, which produced a flat rotation curve. The circular velocity of the halo was taken to be the maximum of the rotation curve. The models are limited in the sense that $\sigma_{z}=\sigma_{r}$ at all places. Whereas this assumption makes it possible to easily calculate models, it is not required by any means that this assumption holds. Full, three integral models are needed for a more extensive exploration of parameter space, but that is too complex for the current purposes. As an alternative, the models were "corrected" for anisotropy as if they were spherical. That is, apparent velocity dispersion profiles were calculated for a spherical model, under different assumptions for the anisotropy $\beta(r)=1-\sigma_{T}^{2}(r) / \sigma_{r}^{2}(r)$. Then "correction" functions $\operatorname{cor}(r, \beta)=\sigma_{o b s}(r, \beta) / \sigma_{o b s}(r$, isotropic $)$ were evaluated, and applied to the axisymmetric models. These corrections should be taken only as an exploration of parameter space, not as exact answers.

\subsubsection{The Tully-Fisher relation for ellipticals}

As a result, we were able to calculate circular velocities for elliptical galaxies. The first question one can ask, is whether ellipticals fall onto the same relation as spirals in the Tully-Fisher diagram, which relates luminosity and circular velocity. Figure 3 shows the relation for our sample of ellipticals, compared to the sample of Pierce and Tully (1988) on spiral galaxies in Virgo and Ursa Major. Figure 3a shows the maximum circular velocities of ellipticals under the assumption that ellipticals do not have halos. It is clear, that spirals and ellipticals are well separated in this diagram, by about $0.7 \mathrm{mag}$. The circular velocities used in Figure $3 \mathrm{~b}$ were derived under the assumption that ellipticals have dark halos, and that they are radially anisotropic in the outer parts $\left[\beta=r^{2} /\left(r^{2}+r_{e}^{2}\right)\right]$. Clearly the correspondence is better, the difference in magnitude is reduced to 0.3 when Pierce and Tully's relation is used, and -0.1 for the relation by Willick (1990). The circular velocities for the last models are reduced, because the halo helps to support the galaxy, and because of the anisotropy. Both effects reduce the circular velocity by $\approx 10 \%$ and $\approx 5 \%$, respectively.

Ellipticals satisfy Pierce and Tully's relation exactly if the circular velocities of ellipticals are related to the central velocity dispersions by $v_{c i r} / \sigma_{c e n}(o b s)=$ 1.27. The anisotropic halo models discussed above produce an average ratio of $v_{c i r} / \sigma_{c e n}(o b s)=1.38$. Thus the circular velocities from the models are $10 \%$ higher 


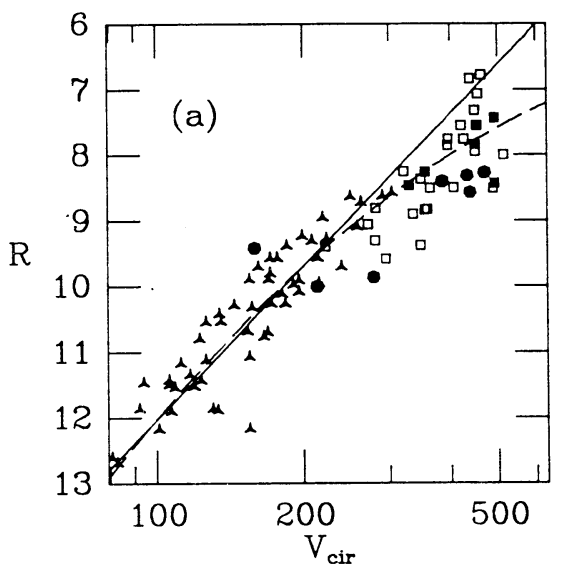

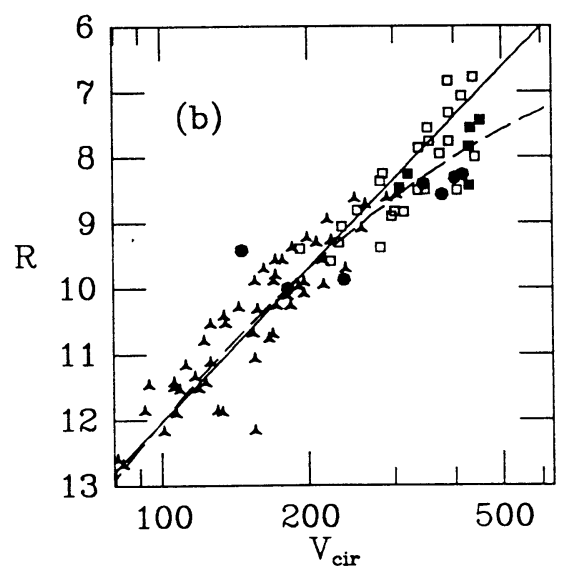

Fig. 3. The R band Tully Fisher relation for spirals in Virgo and Ursa Major (triangles), and ellipticals (boxes and filled symbols). Ellipticals have been rescaled to Virgo distance. Ellipticals in Virgo and Fornax have filled symbols. The drawn line is the Tully-Fisher relation by Pierce and Tully (1988), the dashed line is the relation given by Willick (1990). (a) The ellipticals are assumed not to have halos, and the circular velocity is the maximum circular velocity. (b) The ellipticals are assumed to have halos, and have anisotropic velocity dispersions. The circular velocities for these models are constant in the outer parts. The offset between ellipticals and spirals is rather small for these models.

than the circular velocities expected if ellipticals satisfy the Pierce and Tully's relation exactly.

It is important to note, however, that there is very little overlap in the circular velocities. Most ellipticals have large circular velocities (>250 km/s), whereas most spirals have $v_{c}<250 \mathrm{~km} / \mathrm{s}$. Thus a relation with a different shape will give a different offset. It is obvious that in the area of overlap, the offset between spirals and ellipticals is not very large in Fig. $3 \mathrm{~b}$. The offset reported here is smaller than those reported by Blumenthal et al (1984) and Hernquist and Quinn (1988).

In hindsight, it may not be a surprise that an offset remains between spirals and ellipticals. A residual is expected from the fact that spirals are still forming stars. Even if the baryonic content of a galaxy is uniquely related to the circular velocity, we do not expect star forming galaxies to have exactly the same luminosity as galaxies without star formation. After all, the star forming galaxies will change luminosity with time in a different way as the "dead" galaxies, and we would have been living in a very special epoch if the luminosities would have been a unique function of circular velocity, independent of galaxy type. More models are needed to investigate whether star formation alone can produce the observed differences. Furthermore, there is no obvious reason that the baryonic content is uniquely related to the circular velocity; as a matter of fact, several authors have attempted to explain the Hubble sequence by variations of halo density (and $v_{c}$ ) at constant baryonic mass (e.g., Blumenthal et al 1984, Zurek et al 1988). Finally, it may be 
that the rotation curves of spirals are not exactly flat after all (e.g., Casertano \& van Gorkom 1991). This complicates a direct comparison between different types of galaxies.

\subsubsection{The relation between $v_{c}$ and $\sigma_{c e n}$}

The next question to ask, is how the spheroid is related to the halo. Figure $4 \mathrm{a}$ presents a plot of the circular velocity of the halo versus the central velocity dispersion of the bulge. The data for spirals were taken from Kent $(1986,1988)$, the data for ellipticals were taken from the models described above. Many of the bulges and ellipticals fall along the line $v_{c}=1.38 \sigma_{c e n}$, with a large scatter below the line at low circular velocities.

The main parameter responsible for the scatter below the line may very well be bulge/disk ratio. Figure $4 \mathrm{~b}$ presents the ratio of bulge light to total light ratio $(B / T)$ versus $\sigma_{c e n} / v_{c}$. The ellipticals are taken to have $B / T=1$, and the $B / T$ ratios for spirals were taken from Kent $(1986,1988)$. Note that similar diagrams were published by Whitmore et al (1979), and Whitmore and Kirshner (1981). The left most point is the extrapolated ratio of $\sigma_{c e n} / v_{c}$ for a pure disk, taken from the work of Bottema (see Bottema et al 1991, and references therein). There is an obvious trend, in the sense that galaxies with $\mathrm{B} / \mathrm{T}<0.2$ have a low $\sigma_{c e n} / v_{c}$. The ratio of $\sigma_{c e n} / v_{c}$ for these galaxies is comparable to the mean of $\sigma_{c e n} / v_{c}$ for pure disks. At larger values of $\mathrm{B} / \mathrm{T}$ there may be a weak trend. It is encouraging that the observed values for bulges with $B / T>0.5$ agrees well with the model value for ellipticals.

It is not clear how much of the scatter is observational, and more and better data would be useful. The data are certainly consistent with the idea that $\sigma_{c e n} / v_{c}$ drops systematically with decreasing bulge to disk ratio. This is not required by stellar dynamics, but must be imposed by the formation process (e.g., Faber 1982).

\section{Stellar Populations}

Recent reviews of this subject can be found in other papers in this conference proceedings, and in the proceedings of IAU Symp 149 (Barbuy and Renzini 1992). Here a short review of some topics of interest is given.

\subsection{AGES}

The most relevant quantity one would like to determine of a stellar population, is probably its age. It is notoriously difficult, however, to separate the effects of age differences from the effects of metallicity differences. Broad band colors allow no separation (see, e.g., the results of Arimoto and Yoshii 1987). It had been hoped that absorption line strengths could provide a way to separate age effects and metallicity effects, but even that turns out to be very difficult (e.g., Peletier, 1989, Worthey et al 1992). Detailed analyses of wide band spectrophotometric data find evidence for intermediate age populations (e.g., Pickles, 1985). 

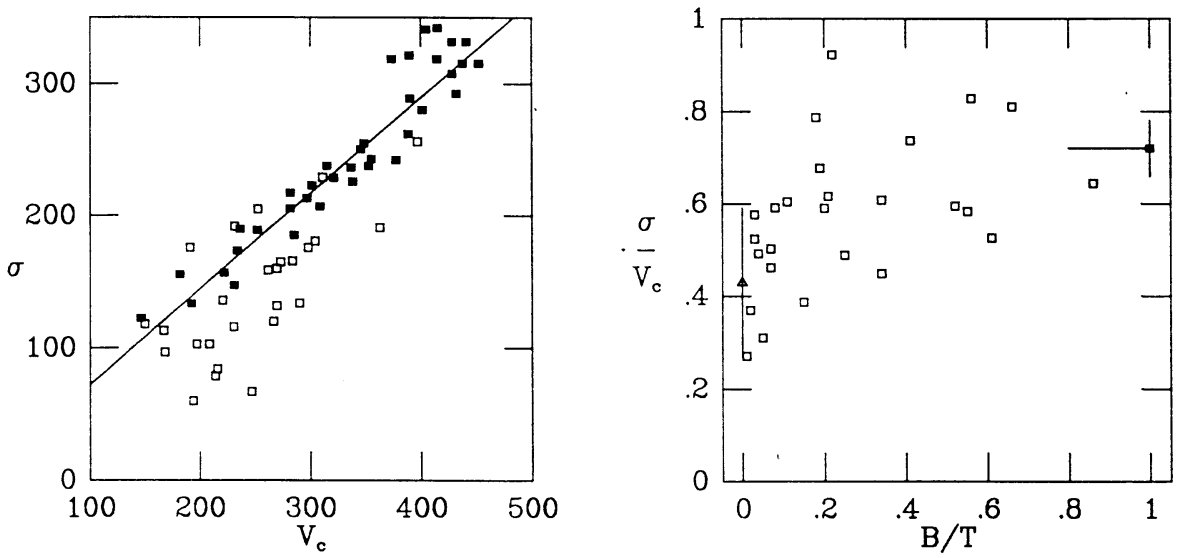

Fig. 4. (a) The central velocity dispersion $\sigma_{c e n}$ against halo circular velocity $v_{c}$. Open symbols are bulges, closed symbols are ellipticals. The circular velocities for ellipticals are the model values from figure $3 \mathrm{~b}$. (b) The ratio of $\sigma_{c e n} / v_{c}$ against the ratio of Bulge to Total light $(\mathrm{B} / \mathrm{T})$. The triangle at left is valid for pure disks, the square at right for ellipticals. Note that systems with low $\mathrm{B} / \mathrm{T}$ have $\sigma_{c e n} / v_{c}$ almost equal to $\sigma_{c e n} / v_{c}$ for disks.

A complicating problem is that elliptical galaxies must contain mixes of stellar populations. In a closed box model of chemical enrichment, it is obvious that low metallicity stars form before high metallicity stars do. Thus, one expects a large range of metallicities in ellipticals, ranging from $[\mathrm{Fe} / \mathrm{H}]=-\infty$ to $[\mathrm{Fe} / \mathrm{H}]=1$ (?). Observations of the galactic bulge have confirmed this prediction. Rich (1988) found a range in metallicities of a factor of 100 in the galactic bulge.

Arimoto and Yoshii (1987) constructed models of populations with a large intrinsic range in metallicity, and calculated broad band colors. They showed that some results change quite drastically when a range in metallicities is used, in contrast to a single metallicity. The author is not aware of independent follow up studies along these lines. It appears that any analysis based on a single metallicity model is somewhat uncertain until a more complete distribution of metallicities has been used.

\subsection{COLOR MAGNITUDE RELATION}

The best known relation is probably the color magnitude relation (Sandage and Visnavathan 1978). The brighter the galaxy, the redder. Since the absorption line strengths increase too with magnitude, this is generally interpreted as a correlation of metallicity with magnitude. Sandage and Visnavathan (1978) concluded that S0's follow the same relation as ellipticals, and that their populations must be very much alike. Larson et al (1980) found that the spread around the relation depends on galaxy type and environment. The S0's and ellipticals in the field have generally higher scatter than ellipticals in rich clusters. Ellipticals in the field are somewhat 
$\mathbf{z}$

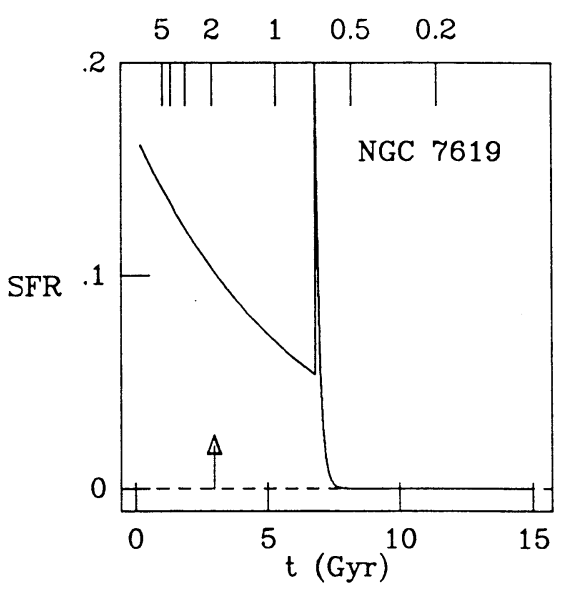

z

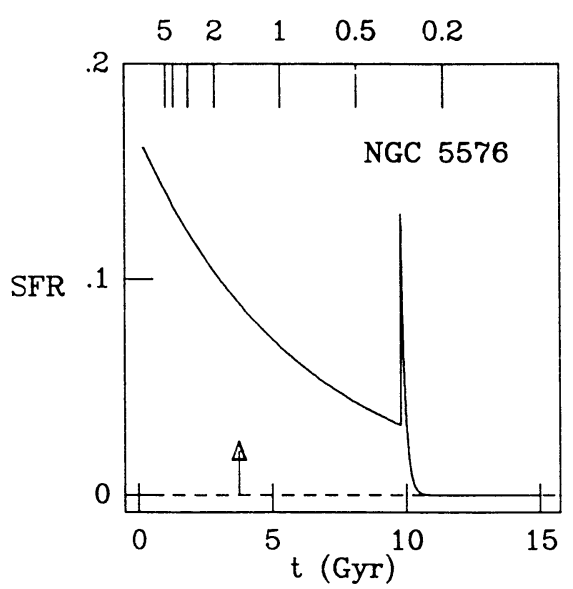

Fig. 5. The star formation histories for ellipticals as modeled by Schweizer \& Seitzer (1992). The galaxies are assumed to evolve like an Sb galaxy, until they merge, and quickly exhaust their gas supply. The two galaxies span a typical range in merger age. Note that merging occurs very late for these systems $\left(0.3<\mathrm{z}<0.7, q_{0}=1 / 2\right)$. The arrows indicate the mean stellar age. The mean stellar is very uniform at $\mathrm{t}=3$ Gyr equivalent to $\mathrm{z} \approx 2$.

bluer than cluster ellipticals. These results indicate that star formation histories probably depend on the environment. More systematic surveys would obviously be useful.

\subsection{THE SPREAD IN MEAN AGES}

The color magnitude relation implies that the scatter in color is quite low, given the magnitude. This can be used to obtain a constraint on the spread in ages. Schweizer and Seitzer (1992) analyzed a sample of field ellipticals. They used deep photometry, and overall colors of galaxies to try to estimate the star formation history of galaxies. Their observed scatter in $U-V$ at a given absolute magnitude was 0.07 mag. Their (merger) models assumed that the star formation decays exponentially in the progenitor galaxies, until a merger occurs, in which part of the remaining gas is quickly converted into stars, and the rest is expelled from the galaxy. A schematic representation of such a formation scenario is given in Figure $5 \mathrm{a}$ and $\mathrm{b}$. The mergers occurred about $8 \pm 2 \mathrm{Gyr}$ ago, and the universe is taken to be $15 \mathrm{Gyr}$ old. This relatively late "assembly" implies that we can observe these mergers at redshifts between 0.3 and 0.7 . The mean stellar age implied by their model is much higher, 12 $\pm 0.5 \mathrm{Gyr}$, corresponding to a redshift of 1.9 . This illustrates clearly that the "epoch of star formation" is not necessarily the same as the "epoch of galaxy assembly", and constraints on the mean ages are not necessarily constraints on the last major event in a galaxies lifetime.

The same spread in colors can be modeled in a completely different way. If it is assumed that the galaxy forms in a very short star burst, then the spread implies a 
spread in stellar age of about 4 Gyr (FWHM). This model implies that enormous star bursts occur in a redshift range of 1.4 to $\infty$, if the bursts start at very high redshift. It may equally well be that metallicity variations add to the scatter (e.g., Bertola et al 1993), or small bursts of starformation at very recent times. It is clear that different models, which reproduce the data equally well, produce very different predictions in redshift space. One may therefore hope that observations of high redshift galaxies will help greatly to solve the ambiguity.

A recent study of Coma and Virgo by Bower et al (1992) produced a spread in the color magnitude diagram of about 0.04 in $U-V$, about half the spread found by Schweizer \& Seitzer (1992) for their field sample. The result implies that the formation history of these cluster galaxies was more uniform. The same ambiguities remain in the modeling of this data.

\section{Summary and Discussion}

The similarity of ellipticals and bulges is striking. Many ellipticals may actually have faint disks, demonstrating that disk formation is an even more common aspect of galaxy formation than previously thought. The exception is formed by very bright elliptical galaxies, which are usually the brightest, or among the brightest galaxies in groups and clusters. There is no strong evidence for disks in these galaxies. Thus, if one defines ellipticals narrowly as galaxies without disks, many, if not most of the ellipticals should be redefined as S0's, and we are left with Bright Cluster Members as "true ellipticals". However, the renaming of (faint) ellipticals into S0's does not explain very much, and is certainly not favored by the author.

Thus, whereas disk formation may be a common process in the formation of ellipticals, or, generally speaking, high Bulge/Disk ratio systems, bulge formation may be part of the disk formation in low B/D systems. At the lowest B/D ratios, the bulge velocity dispersions are possibly coupled to the disk velocity dispersions, and the bulges may have formed as part of the formation of the disks.

The available data is consistent with the hypothesis that high $\mathrm{B} / \mathrm{D}$ systems have halos, but the evidence is still scarce. An exploration of models indicates that ellipticals may follow more or less the same relation between luminous mass and halo circular velocity, but this result remains model dependent. The ratio of bulge velocity dispersion to halo circular velocity decreases with decreasing B/D ratio. This must be imposed by the formation process.

The question to be answered at this stage is what determines the Bulge/Disk ratio of galaxies, and the correlation between $\mathrm{B} / \mathrm{D}$ ratio and environment and luminosity. It is obvious that baryonic processes (like dissipation, star formation, reheating, etc.) play an important role. Dissipationless collapse would produce rather similar halos, and without the systematic changes that are observed in the baryonic components (e.g., Barnes and Efstathiou 1987). Nevertheless, since the dark matter contributes most of the mass, it must play an important role in determining the structure of the galaxies. Possibly the most essential question that can be asked at this moment is how the luminous parts of galaxies are related to their halos. The current evidence suggests that early type systems have slightly higher circular velocities for the same red luminosity. The difference may only be small, however, (15\% 
?), and is partly or fully caused by different $\mathrm{M} / \mathrm{L}$ ratios, and systematic changes in the shapes of rotation curve with mass.

Zurek et al (1988), and Katz (1992) speculated that the Hubble type was driven by the properties of the individual (dark matter) clumps before assembly. A higher differentiation of clumps before assembly can lead to higher cooling rates, and higher star formation rates before the assembly. During the assembly (or "merger") the more differentiated clumps can loose more angular momentum against the dark halo material. This explains why the specific angular momentum decreases with earlier Hubble type. The fact that early type galaxies may have higher halo densities (as demonstrated by their higher circular velocities) is in agreement with this hypothesis.

\subsection{FUTURE WORK}

The numerical simulations of galaxy formation in CDM type universes are maturing at this very moment. Previously, questions about details of the baryonic component of galaxies could not be addressed, but now, with the inclusion of hydrodynamics, such questions can actually be studied (e.g., Katz et al 1992). It appears as if much progress can be made along these lines. The current results are very encouraging, but, of course, one cannot claim to have understood all properties at this moment. Feedback processes like heat injection from supernovae in the ISM, and the role of nuclear activity have not been fully explored. The total amount of energy released by both phenomena is very large, and must be important in the overall galaxy formation process.

On the observational side, there is a need for systematic studies of the dark matter components of galaxies, and their relation with the luminous parts. It is important to try to understand the relation between galaxy morphology/structure versus halo structure. Unfortunately, such studies are very difficult for galaxies other than late type spirals, and they will require large investments of telescope time. Studies. of systematic changes of galaxy properties with environment (or mean density) are extremely useful. It should not come as a surprise, if the galaxy formation process is significantly influenced by the underlying, large scale density distribution (e.g., Katz et al, 1992).

Secondly, direct observations of galaxies at high redshift are becoming possible, which enables us to observe some of the processes that are speculated to occur. An interesting example are the high redshift radio galaxies, where star formation appears to be coupled to the nuclear activity (e.g., Chambers et al 1990 ). Another example is the detection of evolution of galaxies in rich clusters (" the ButcherOemler effect", Butcher \& Oemler 1984). Studies of such galaxies may provide a glimpse of the formation process in working.

\section{Acknowledgements}

It is a pleasure to thank the organizers of the conference for their support. Discussions with Steve Kent, Neal Katz, Hans-Walter Rix, and Paul Schechter are gratefully acknowledged. 
Tim de Zeeuw and Konrad Kuijken commented on the manuscript. This research has been funded by Hubble Fellowship grant HF-1016.01.91A.

\section{References}

Arimoto, N., \& Yoshii, Y. 1987, AA, 173, 23.

Athanassoula, E., 1983, IAU Symposium 100 "Internal Kinematics and Dynamics of Galaxies" (Dordrecht: Reidel).

Barnes, J., \& White, S. D. M., 1984, MNRAS, 211, 253.

Barnes, J., \& Efstathiou, G., 1987, ApJ, 319, 575.

Barbuy, B., \& Renzini, A., IAU symposium 149 "The Stellar Populations of Galaxies", (Dordrecht: Kluwer).

Bender, R., 1988a, $A A, 193, \mathrm{~L} 7$.

Bender, R., 1988b, $A A, 202$, L5.

Bender, R., Burstein, D., \& Faber, S. M., 1992, ApJ, 399, 462.

Bender, R., Döbereiner, S., \& Möllenhof, C., 1988, A AS up, 74, 385.

Bender, R., \& Nieto, J. L., 1990, AA, 239, 97.

Bertola, F., Burstein, D., \& Buson, L., 1993, ApJ, 403, 573.

van den Bergh, S., 1990, ApJ, 348, 57.

Binggeli, B., Sandage, A., \& Tammann, G. A., 1988, ARAA, 26, 509.

Binney, J. J., \& Manon, G. A., 1982, MNRAS, 200, 361.

Blumenthal, G. R., Faber, S. M., Primack, J. R., \& Rees, M. J., 1984, Nature, 311, 517.

Bottema, R., van der Kruit, B. \& Valentijn, E., 1991, AA, 247, 357.

Bower, R. G., Lucey, J. R., \& Ellis, R. S., 1992, MNRAS, 254, 601.

Broeils, A. H., 1992, PhD Thesis, University of Groningen.

Butcher, H., \& Oemler, A., 1984, ApJ, 285, 426.

Carter, D., 1978, MNRAS, $182,797$.

Carter, D., 1987, ApJ, 312, 514.

Capaccioli, M., 1987, in IAU symposium 127, "Structure and Dynamics of Elliptical Galaxies", ed. T. de Zeeuw (Dordrecht: Reidel), p47.

Capaccioli, M., Caon, N., \& D'Onofrio, M. 1992, MNRAS, 259, 323.

Casertano, S., \& van Gorkom, J. H., 1991, AJ, 101, 1231.

Chambers, K. C., Miley, G. K., \& van Breugel, W. J. M., 1990, ApJ, 363, 21.

de Zeeuw, P. T., 1987, IAU symposium 127, "Structure and Dynamics of Elliptical Galaxies", (Dordrecht: Reidel).

de Zeeuw, P. T., 1992, in "Morphological and Physical Classification of Galaxies", ed. Busarello et al (Dordrecht: Kluwer).

de Zeeuw, P. T., \& Franx, M, 1991, ARAA, 29, 239.

Davies, R. L., Efstathiou, G., Fall, S. M., Ilingworth, G. D., \& Schechter, P. L., 1983, ApJ, 266, 41.

Dressler, A., 1980, ApJ, 236, 351.

Dressler, A., 1984, ApJ, 286, 97.

Dressler, A., 1987, ApJ, 317, 1.

Dressler, A., \& Sandage, A. 1983, ApJ, 265, 664.

Efstathiou, G., Ellis, R. S., \& Carter, D., 1982, MNRAS, 201, 975.

Faber, S. M., 1982, in "Astrophysical Cosmology", ed. H.A. Brück et al (Vatican: Pontificia Academia Scientiarum), p219.

Faber, S. M., Dressler, A., Davies, R. L., Burstein, D., Lynden-Bell, D., Terlevich, R. J., \& Wegner, G., 1987, in "Nearly Normal Galaxies", ed. S. M. Faber (New York: Springer), p. 175

Forman, W., Jones, C., \& Tucker, W., 1985, ApJ, 293, 102.

Fisher, D., Mlingworth, G. D., \& Franx, M., 1993, in preparation.

Franx, M., \& Mlingworth, G. D., 1988, ApJ (Letters), 327, 55.

Franx, M., Illingworth, G. D., \& Heckman, T., 1989, ApJ, 344, 613.

Franx, M., Illingworth, G. D., \& de Zeeuw, P. T., 1991, ApJ, 383, 112.

Hernquist, L., \& Quinn, P. J., 1987, ApJ, 312, 1.

Hubble, E., 1936, "Realm of Nebulae" (New Haven: Yale University Press).

Illingworth, G. D., 1983, in IAU Symposium 100, "Internal Kinematics and Dynamics of Galaxies", ed. E. Athanassoula (Dordrecht: Reidel), p257. 
Jedrzejewski, R., \& Schechter, P. L., 1988, ApJ (Letters), 330, 87.

Katz, N., 1992, PASP, 104, 852.

Katz, N., Hernquist, L., \& Weinberg, D. H., 1992, ApJ (Letters), 399, 109.

Kent, S. M., 1981, ApJ, 245, 805.

Kent, S. M., 1984, ApJSup., 56, 105.

Kent, S. M., 1986, $A J, 91,1301$.

Kent, S. M., 1988, $A J, 96,514$.

Kent, S. M., 1990, in ASP conf ser. 10, "Evolution of the Universe of Galaxies", ed. R. G. Kron (Provo: Brigham Young Univ.), p109.

Kormendy, J., \& Пlingworth, G. D., 1982, ApJ, 256, 460.

Kormendy, J., 1988, ApJ, 335, 40.

Kormendy, J., 1982, in "Morphology and Dynamics of Galaxies", ed. L. Martinet \& M. Mayor (Sauverny: Geneva Obs.), p113.

Larson,, R. B., Tinsley, B. M., \& Caldwell, C. N., 1980, ApJ, 237, 692.

Merritt, D. 1988, in ASP conf. ser. 5, "The Minnesota Lectures on Clusters of Galaxies and Large Scale Structure", ed. J. M. Dickey (Provo: Brigham Young Univ.), 175.

Peletier, R. F. P., 1989, PhD Thesis, University of Groningen.

Pierce, M.J., \& Tully, R.B., 1988, A pJ, 330, 579.

Pickles, A. J., 1985, ApJ, 296, 340.

Rich, R. M., 1988, $A J, 95,828$.

Rix, H. W., Franx, M., Fisher, D., \& Illingworth, G., 1992, ApJ (Letters), 400, 5.

Rix, H. W., \& White, S. D. M., 1990, ApJ, 362, 52.

Rubin, V. C., Graham, J. A., \& Kenney, J. D. P., 1992, ApJ (Letters), 394, 9.

Sandage, A., \& Visnavathan, N., 1978, ApJ, 225, 742.

Saglia, R. P., Bertin, G., Bertola, F., Danziger, J., Dejonghe, H., Sadler, E. M., Stiavelli, M., de Zeeuw, P. T., \& Zeilinger, W. W., 1993, ApJ, 403, 573.

Schechter, P. L., \& Dressler, A., 1987, $A J$, 94, 563.

Schweizer, F., \& Seitzer, P. 1992, AJ, 104, 1039.

Trinchieri, G., Fabbiano, G., \& Canizares, C. R., 1986, ApJ, 310, 637.

van der Marel, R. P. 1992, MNRAS, 253, 710.

Whitmore, B. C., Kirshner, R. P., \& Schechter, P. L., 1979, ApJ, 234, 68.

Whitmore, B. C. \& Kirshner, R. P., 1981, ApJ, 250, 43.

Willick, J.A., 1990, ApJ (Letters), 351, L5.

Worthey, G., Faber, S. M., \& Gonzales, J. J., 1992, ApJ, 398, 69.

Zurek, W. H.,Quinn, P. J., \& Salmon, J. K., 1988, ApJ, 330, 519.

\section{DISCUSSION}

Ferguson: The exercise with the colours is an interesting one, but has yet to be subjected to the sort of tests that the Yoshii and Arimoto models have been, with lots of different colours and in predicting the $\mathrm{C}-\mathrm{M}$ diagram and so on. It seems that if the simple chemical evolution models work, they are the Occam's razor solution and the merger models need to be very convincing to argue that they are a significant factor in elliptical formation in general.

Franx: You can separate two things, merging and star formation, and they are not necessarily the same thing of course. My impression is that it will be very hard to distinguish between those two options in the nearby galaxies. When star formation has stopped for $5 \mathrm{Gyr}$, it becomes very hard to say very much about what happened before that, and if you look in redshift space those differences become irrelevant. 
Gerhard: A comment on the determination of the halo in ellipticals: I don't think that the anisotropy problem is really such a big one. I know it has been around for 10 years and it is an effect, but once you go out into the regimes where you actually expect some effect of the halo, I think that the effect of tangential anisotropy, which is one that could mimic dark matter, relative to isotropy is only $20 \%$ or so. When it comes to these differences, I don't think you would be bold enough to interpret this as a heavy halo anyway. The biggest problem is to go out to large enough radii, where you can collect sufficient photons, which is the topic of an ESO key project.

Franx: I think you are right and we can improve on what has been done. We want to settle more than just the question whether elliptical galaxies are very large; we want to determine what their circular velocities are. It still remains to be seen how good you can do that with kinematic data from the light of the stars.

Gerhard: You were showing some rather interesting cases for ellipticals and bulges with sub-structure, and, yes, you can argue that you wouldn't see that structure in a lot of cases, and we could say that in a third of cases you would definitely not see it, but isn't it none the less true that the majority of them are rather boring in a sense?

Franx: Yes, I completely agree with you that we only see this structure in a small fraction of ellipticals. The most amazing aspect is though, that all the other properties of these ellipticals appear to be very normal, so it suggests but doesn't prove in any way, that this kind of sub-structure that we are seeing is one of the many outcomes of the formation process.

Renzini: The recent paper by Bowers, Lucy and Ellis, does much better than Schweizer et.al.: they have a dispersion in colour of only 0.03 magnitude for Virgo and Coma ellipticals, for a given central velocity dispersion. This allows them to set a lower limit to the age of these elliptical galaxies' stellar populations, which is at least 13 Gyr for over $90 \%$ of the light we see today. So, at this point it is important to get it through to people that when you compare the so called fast formation process for ellipticals to a slow formation process, we have the two time scales which are at this point constrained to be less than just a few Gyr. That has not been, in clusters, a continuous formation of ellipticals at a nearly constant rate over the last 15 Gyr, as a result of merging.

Franx: It is well known effect, that when we look at clusters of galaxies at intermediate redshifts, they appear to be full with old galaxies. I showed a cluster at a redshift of only 0.2 , but when we make a colour map of that cluster, it is indeed full of old galaxies, but it is well known that even at these redshifts we see some indications of blue galaxies. This is of course the Butcher-Oemler effect, which has been observed by many other people as well. It actually turns out that it is possible to derive rotation curves for these galaxies and in the cluster I showed, the largest blue galaxy is rotating very rapidly. So what this galaxy probably is, is an S0 galaxy in transformation. So we shouldn't lose sight that, despite the constraints of newer 
data, even at very low redshifts we do see evidence of star formation and galaxy transformation.

Renzini: Okay, one can always find weird objects, but one must quantify statements instead of making an example of one case, no matter how many such weird cases there are. I wanted to make a second point, you have used so far only the dispersion in the $\mathrm{C}-\sigma$ relation, there is the trend to explain as well. Galaxies with higher central velocity dispersion, which means a deeper potential well, are redder and this to me means you have to form the stars within that particular potential. You don't merge already formed stellar systems. You merge, if you do merge, gas and then you make the stars.

Franx: The CDM simulations (in the literature) show that the depth of a perturbation at redshift zero is closely correlated with the depth of the perturbations of which they are formed from at a redshift of 1.0, so there is a very good correlation between the halos before they merge and after they merge. Which is probably only saying that big clumps form even bigger clumps.

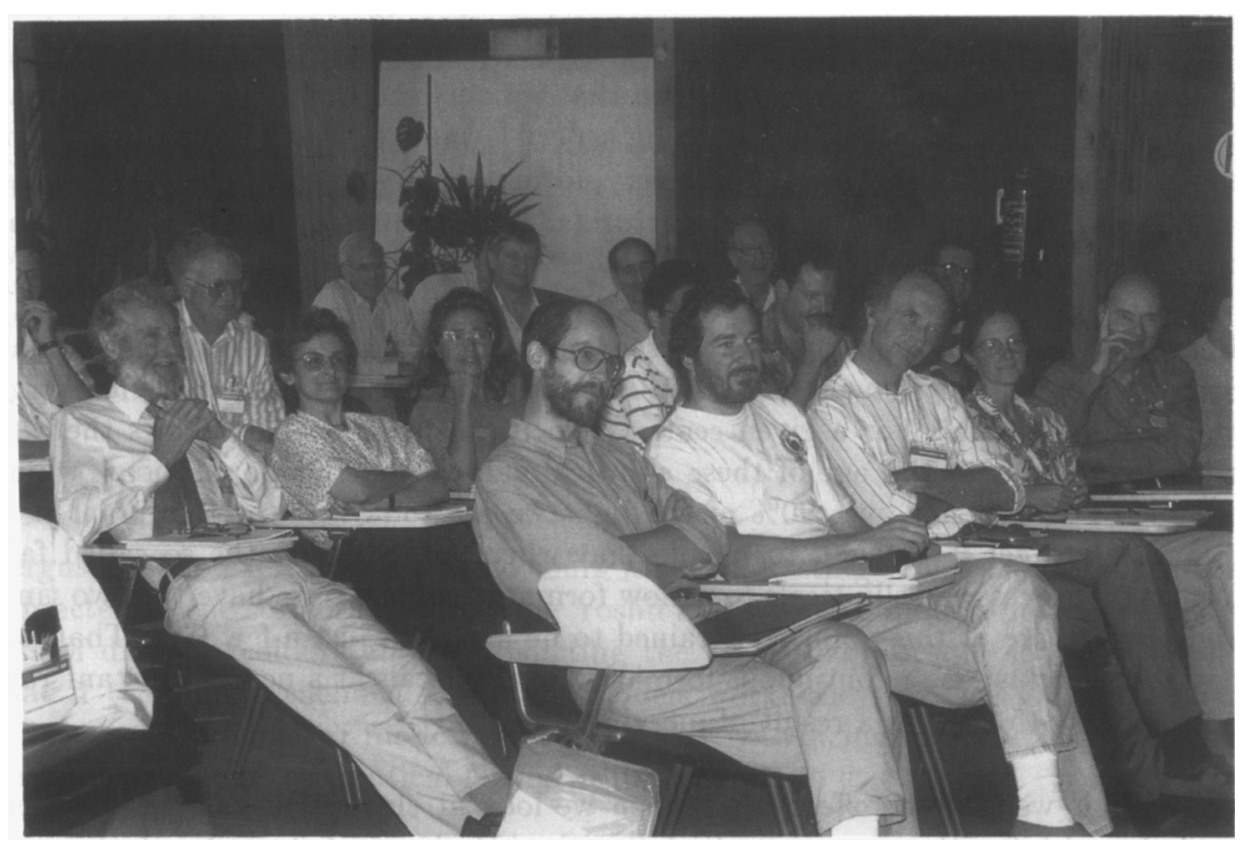

The artist, L. Debrouwere (first in front row), enjoying a rare moment of rest during Habing's concluding words 


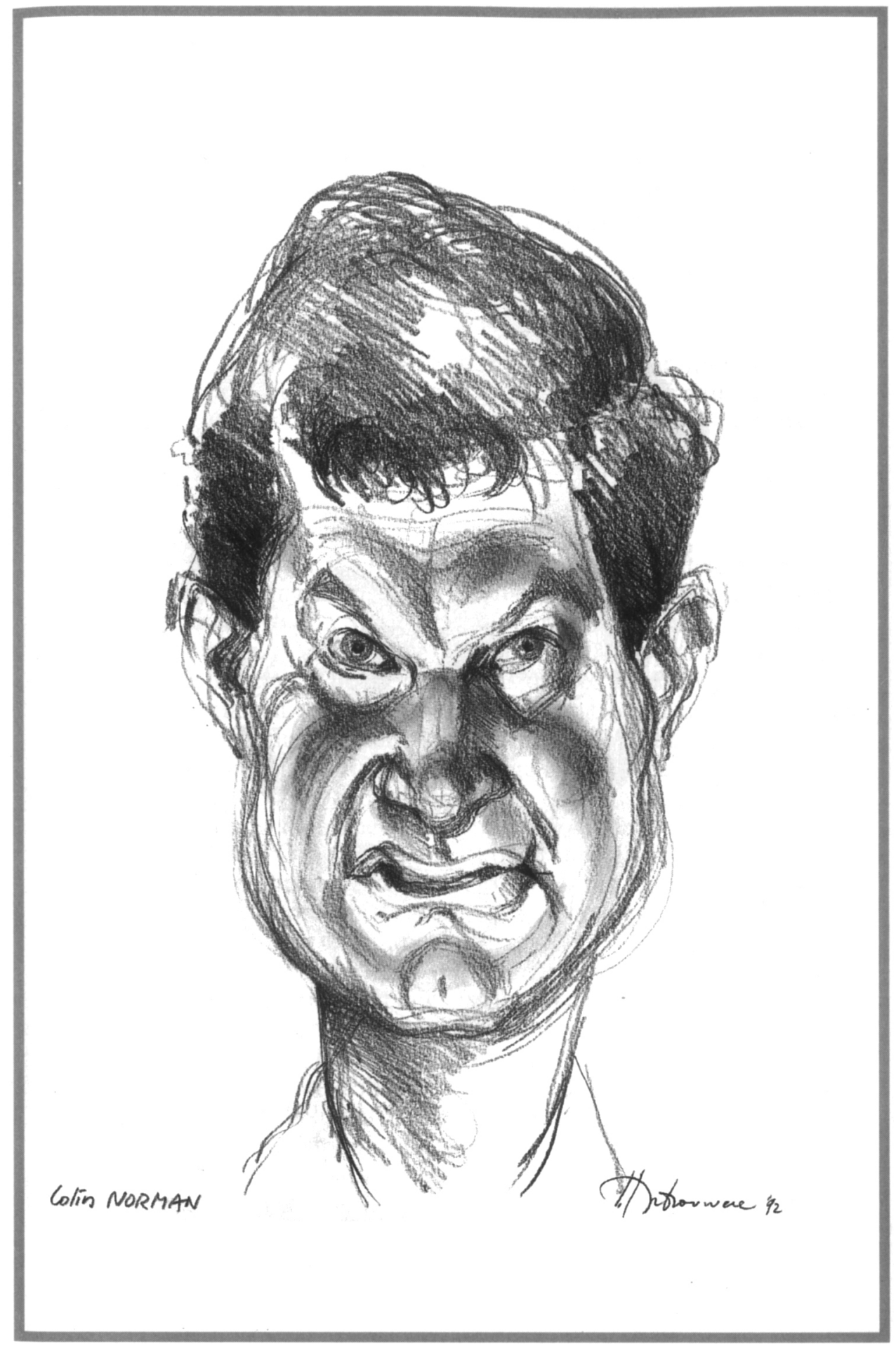




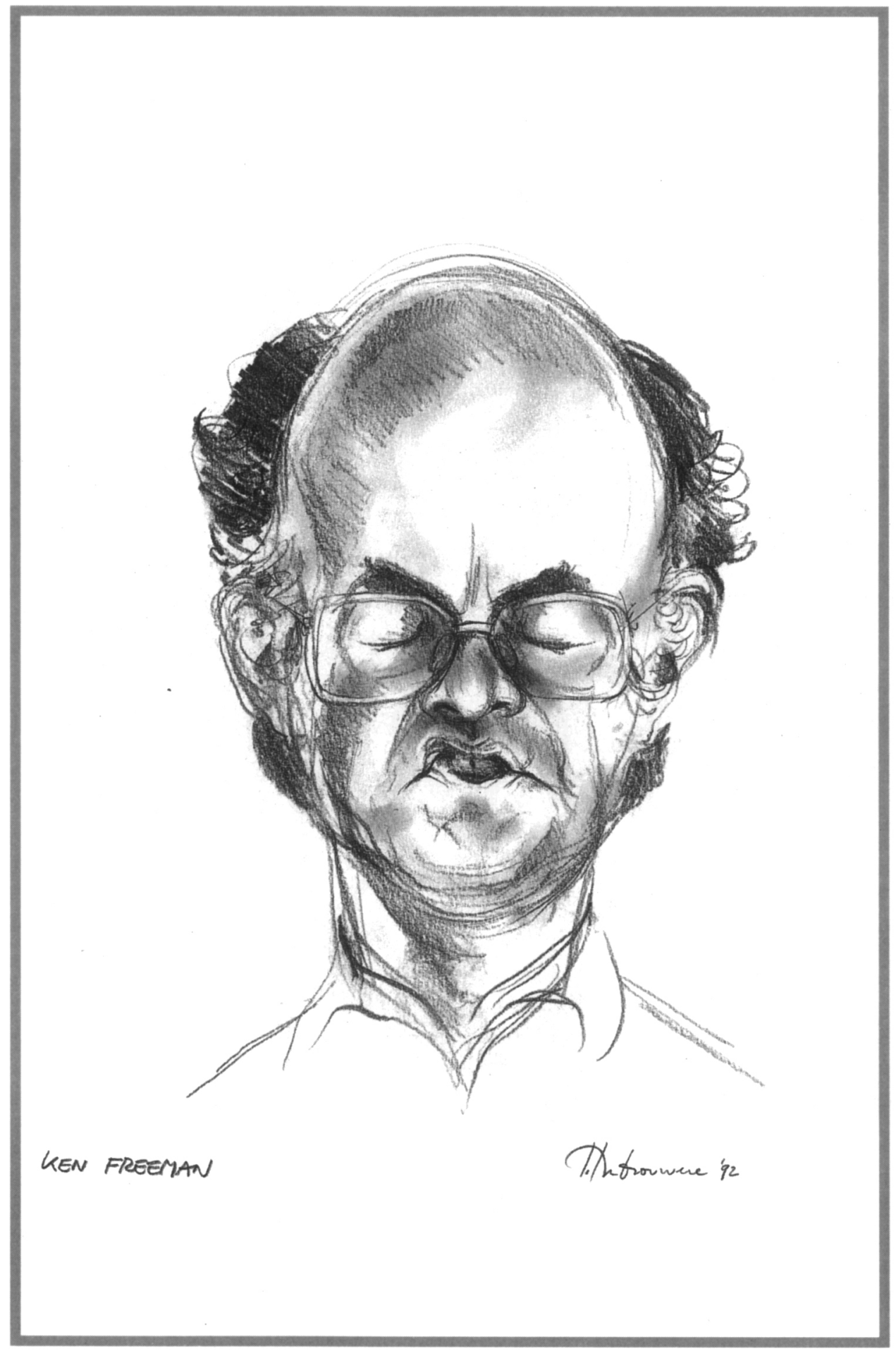

